ISSN 1997-342X (Online), ISSN 1991-8631 (Print)

Original Paper http://ajol.info/index.php/ijbcs http://indexmedicus.afro.who.int

\title{
Détermination des doses optimales de fumures organo-minérales en microdose dans la zone soudano-sahélienne du Burkina Faso
}

\author{
Béatrice B. SOMDA ${ }^{1 *}$, Badiori OUATTARA ${ }^{1}$, Idriss SERME ${ }^{1}$, Mathias B. POUYA ${ }^{2}$, \\ François LOMPO ${ }^{1}$, S. Jean Baptiste TAONDA ${ }^{1}$ et P. Michel SEDOGO ${ }^{1}$
}

\author{
${ }^{1}$ Institut de l'Environnement et de Recherches Agricoles (INERA), Burkina Faso. \\ ${ }^{2}$ Réseau MARP, Burkina Faso. \\ ${ }^{*}$ Auteur correspondant ; E-mail: beatricesomda@yahoo.fr
}

\begin{abstract}
REMERCIEMENTS
Nous adressons nos sincères remerciements au projet micro-dose du Centre De RechercheDéveloppement International (CRDI) pour avoir financé cette étude.
\end{abstract}

\section{RESUME}

La technique de fertilisation minérale par micro-dose a montré son efficacité pour l'amélioration de la productivité agricole en Afrique Sub-saharienne. Cependant, celle-ci n'a toujours pas été associée de façon optimale à la fumure organique. L'objectif de cette étude est de déterminer les doses optimales de matière organique et d'engrais minéraux combinés en microdose, les dates optimales d'apport des engrais minéraux et leurs effets sur quelques caractéristiques chimiques du sol et sur la production agricole. L'étude a été conduite en vase de végétation selon un dispositif split-split plot. Trois niveaux de fumure organique $\left(0 \mathrm{tha}^{-1}, 5 \mathrm{t} \mathrm{ha}^{-1} \mathrm{et}\right.$ $\left.10 \mathrm{t} \mathrm{ha}^{-1}\right)$ ont été combinés à cinq doses d'engrais minéral $(0 \mathrm{~g}, 1,5 \mathrm{~g}, 2 \mathrm{~g}, 2,5 \mathrm{~g}$ et $3 \mathrm{~g}$ par poquet $)$ en quatre répétitions sur le fonio et le sorgho. Les engrais minéraux ont été apportés à 0,10 et 15 jours après semis. Les résultats obtenus montrent que les doses optimales des fumures sont de $2 \mathrm{~g} \mathrm{poquet}^{-1}$ (soit $62,5 \mathrm{~kg} \mathrm{ha}^{-1}$ ) pour l'engrais minéral et de $5 \mathrm{t} \mathrm{ha}^{-1}$ pour le fumier. Cette combinaison permet de tripler les rendements de biomasse, comparativement au témoin absolu. L'application des engrais minéraux à différents moments n'a pas affecté les rendements de façon significative. Le $\mathrm{pH}$ du sol a été rehaussé d'environ une unité et de plus d'une unité respectivement sous 5 et $10 \mathrm{tha}^{-1}$ de fumier. Les teneurs en matière organique (MO) qui étaient inférieures à $1 \%$ sur les parcelles à fertilisation minérale exclusive sont passées à $1,08 \%$ et $1,26 \%$ respectivement lorsqu'on apporte 5 tha $^{-1}$ et $10 \mathrm{t} \mathrm{ha}^{-1}$ de MO. Les teneurs en phosphore assimilable ont augmenté de 21 et $34 \%$ avec la fertilisation organo-minérale ( $5 \mathrm{tha}^{-1}$ et $10 \mathrm{tha}^{-1} \mathrm{de} \mathrm{MO}$ respectivement) comparativement aux traitements à engrais minéraux exclusifs.

(C) 2017 International Formulae Group. All rights reserved.

Mots clés: Apports combinés, fertilisation minérale, matière organique, fonio, sorgho.

\section{INTRODUCTION}

La faible teneur des sols en éléments nutritifs est constamment évoquée comme étant l'une des contraintes majeures à la production agricole en Afrique Subsaharienne (Lahmar et al., 2011). En effet, ces sols peu profonds ont une faible capacité de rétention en eau et une disponibilité limitée en 
nutriments (Lahmar et al., 2011). Au Burkina Faso, les teneurs en azote et en phosphore de la majeure partie des sols sont inférieures à 0,06\% (UA/SAFGRAD, 2010).

En outre, la dégradation de ces sols suite à des phénomènes naturels (érosion hydrique et/ou éolienne) et/ou anthropiques contribue largement à les appauvrir davantage, rendant ainsi aléatoire leur productivité. Plus de $24 \%$ des sols cultivables sont affectés par ce phénomène (UA/SAFGRAD, 2010).

Par ailleurs, les engrais minéraux et la matière organique qui constituent d'importantes sources d'éléments nutritifs pour les plantes sont très peu utilisés. En effet, la dose d'apport des engrais minéraux est inférieure à $10 \quad \mathrm{~kg} \quad \mathrm{ha}^{-1} \mathrm{en}$ Afrique Subsaharienne (Buerkert et al., 2001; Bado, 2002 ; Morris et al., 2007 ; Bagayoko et al., 2011). Au Burkina Faso, elle a été de $10,95 \mathrm{~kg}$ ha $^{-1}$ en 2003 contre 9,13kg ha-1 en 2009 (MAFAP, 2013). Quant à la fumure organique, elle est apportée sur $33,4 \%$ des superficies cultivées en 2008 contre $21,6 \%$ en 2010 (MAFAP, 2013). Ces apports insuffisants et en baisse se justifieraient par l'indisponibilité de la matière organique, les coûts prohibitifs des engrais minéraux et les faibles coûts des produits agricoles (Bado, 2002 ; MASSE, 2007; Bagayogo et al., 2011).

Face au faible pouvoir d'achat des producteurs, des techniques de fertilisation accessibles et susceptibles d'accroître les rendements, tout en maintenant la fertilité des sols à long terme devraient être développées (Bado, 2002). C'est ainsi qu'un certain nombre de paquets technologiques telle l'adjonction de la fumure organique à la fumure minérale a été développé. L'efficacité agronomique et les effets améliorateurs de la fertilité des sols par cette pratique ne sont plus à démontrer. Malheureusement, l'accessibilité à ces intrants demeure un frein à la mise en œuvre effective de cette technologie par les petits producteurs. En outre, le mode d'épandage des engrais (à la volée) préconisé par les institutions de recherche ne permet pas aux cultures de bénéficier au maximum du peu de nutriments apportés.

Aussi, la technologie de fertilisation minérale par micro-dose ou fertilisation localisée au poquet avec de faibles doses d'engrais a été mise au point comme une alternative aux coûts des engrais (Buerkert et al., 2001 ; Hayashi et al., 2007 ; Bagayogo et al., 2011). Cette technologie s'est avérée performante en termes de productivité sur différents types de sol et de cultures. Toutefois, les investigations en la matière se sont davantage focalisées sur le microdosage des engrais minéraux exclusifs (Tabo et al., 2006). La présente étude vise principalement la détermination des doses optimales combinées de matière organique et d'engrais minéraux par micro-dose sur la production. Il s'agit spécifiquement de: déterminer les doses optimales de fertilisants minéraux par microdose associés à la matière organique sur la production, déterminer les dates optimales d'apport des engrais minéraux par micro-dose et d'évaluer les effets induits sur les caractéristiques chimiques du sol après culture

\section{MATERIEL ET METHODES Matériel d'étude}

L'étude a été menée en vase de végétation au Centre de Recherches Environnementales, Agricoles et de Formation (CREAF) de Kamboinsé, situé à 13 kilomètres au Nord de la ville de Ouagadougou. Le fonio (Digitaria exilis): provenant de la" Collection Voltaïque du Fonio" (CVF) et le sorgho (Sorghum bicolor): variété Sariasso 11 ont été utilisés comme plantes tests. Les fertilisants apportés sont le fumier bien décomposé, l'engrais minéral NPK (14-23-14-3B-1S) et l'urée $(46 \% \mathrm{~N})$.

Le sol utilisé est celui de Saria, un lixisol ferrique. Ce type de sol est pauvre en phosphore assimilable $\left(\approx 15 \mathrm{mg} \mathrm{kg}^{-1} \mathrm{de}\right.$ sol), en azote $\left(\approx 0,7 \mathrm{~g} \mathrm{~kg}^{-1}\right.$ de sol) et en matière organique $(\leq 1 \%)$ avec une faible capacité de rétention en eau allant de 80 à $100 \mathrm{~mm} / \mathrm{m}$ (Barro et al., 2009). Il représente 39\% des sols du Burkina Faso (Soma, 2010). C'est un des 
types de sol majoritaire du pays sur lesquels le sorgho et le fonio sont produits. Les échantillons de sol ont été prélevés à la profondeur de $0-20 \mathrm{~cm}$ à différents points d'un champ paysan. Saria est situé au CentreOuest du Burkina Faso avec pour coordonnées géographiques $12^{\circ} 16^{\prime} \mathrm{N}$ de latitude, $2^{\circ} 9^{\prime}$ ' W de longitude et $300 \mathrm{~m}$ d'altitude.

\section{Dispositif expérimental}

Deux dispositifs expérimentaux ont été mis en place. Un dispositif en split-split plot combinant 3 doses de matières organiques à 5 doses de NPK appliqué à trois périodes (jour du semis, 10 et 15 jours après semis) sur le fonio. La matière organique constitue le facteur principal (blocs), le NPK représente le facteur secondaire dans les sous parcelles et la date d'application du NPK le facteur tertiaire sur les parcelles élémentaires. Le second dispositif en split plot utilise également les 3 doses de MO sur les parcelles principales et les 5 niveaux de NPK sur les parcelles élémentaires au niveau du sorgho. Les pots utilisés pour l'essai étaient de $150 \mathrm{~cm}^{3}$ et contenaient $180 \mathrm{~g}$ de sol tamisé à $0,5 \mathrm{~mm}$ pour le fonio et de 15 litres contenant $17,5 \mathrm{~kg}$ de sol pour le sorgho. Les traitements et les doses de fertilisants apportées dans les pots ensemencés en fonio sont consignés dans le Tableau 1. Pour le sorgho chaque pot a été considéré comme un poquet. L'essai a duré 50 jours pour le fonio et 105 jours pour le sorgho. Juste la biomasse a été considérée au niveau $\mathrm{du}$ fonio tandis que le sorgho a été conduit jusqu'à maturité.

\section{Entretien des cultures}

Le sorgho a été semé à l'aide d'une pioche à quatre graines au maximum par poquet puis démarié à trois plants. Au niveau $\mathrm{du}$ fonio, environ vingt-cinq graines ont été épandues dans le pot puis recouvertes d'une fine couche de sol. L'irrigation a été faite trois fois par jour de sorte que l'eau ne constituait pas un facteur limitant pour la production. Les mauvaises herbes ont été éliminées par binage toutes les deux semaines.

\section{Paramètres mesurés}

$\mathrm{Au}$ bout des 50 jours, la biomasse aérienne du fonio a été fauchée pot par pot et les racines prélevées dans chacun des pots. Chacune de ces biomasses a été séchée à l'étuve à $60{ }^{\circ} \mathrm{C}$ pendant $72 \mathrm{~h}$ puis pesée pour obtenir le poids par pot. Le sorgho a été récolté 105 jours après le semis. Les panicules et la paille ont été séchées à l'air libre pendant deux semaines puis pesées. Les sols après culture ont été broyés et tamisés à 0,5 et à 0,2 $\mathrm{mm}$ pour les analyses chimiques qui ont concerné le $\mathrm{pH}_{\text {eau, }}$ le carbone organique et le phosphore assimilable.

\section{Méthodes d'analyses chimiques}

Le $\mathrm{pH}$ eau du sol a été mesuré à l'aide de la méthode électrométrique dans un rapport sol/eau de 1/2,5. La méthode consiste à introduire dans un flacon $20 \mathrm{~g}$ de sol tamisé à $0,5 \mathrm{~mm}$ et à y ajouter $50 \mathrm{ml}$ d'eau distillée. Le mélange obtenu est agité pendant une heure conformément à la norme AFNOR (1981). Le $\mathrm{pH}$ a été directement lu sur le $\mathrm{pH}$-mètre HANNA à électrode en verre.

Le carbone organique du sol a été dosé selon la méthode de Walkley- Black (Walkley et Black, 1934). C'est une oxydation à froid du carbone du sol à l'aide du bichromate de potassium $\left(\mathrm{K}_{2} \mathrm{Cr}_{2} \mathrm{O}_{7}\right) 1 \mathrm{~N}$ dans un milieu acide sulfurique $\left(\mathrm{H}_{2} \mathrm{SO}_{4}\right)$ concentré. La quantité de bichromate de potassium réduite est proportionnelle à la teneur en carbone du sol. L'excès de bichromate a été titré par le sel de Mohr 0,5 $\mathrm{N}\left[\mathrm{Fe}\left(\mathrm{SO}_{4}\right)_{2}\left(\mathrm{NH}_{4}\right)_{2}\right]$ en présence d'un indicateur coloré (la phénophtaléine). Le pourcentage $\mathrm{du}$ carbone $\mathrm{du}$ sol a été déterminé par la formule suivante:

$\mathrm{C}(\%)=((\mathrm{V} 1-\mathrm{V} 2) \times \mathrm{N} \times \mathbf{0 , 3} \times \mathbf{1 , 3 3}) / \mathrm{P}$

$\mathrm{V} 1$ et V2 désignent les volumes de sel de Mohr utilisés respectivement pour le blanc (sans

Carbone) et pour l'échantillon ; $\mathrm{N}=$ normalité du sel de Mohr et $\mathrm{P}=$ prise d'essai.

Les taux de matière organique et de carbone ont été calculés par les formules suivantes : 
MO $(\%)=[(\mathbf{P i}-\mathbf{P f}) / \mathrm{Pi}] \mathbf{X} 100$ et $\mathrm{C}(\%)=$ MO (\%)/ 1,724

avec: $\mathrm{Pi}=$ le poids initial de la prise d'essai ; $\mathrm{Pf}=$ le poids final de la prise d'essai après calcination.

Le $\mathrm{P}$ assimilable $\left(\mathrm{P}_{\text {ass }}\right)$ est extrait selon la méthode Bray I (Bray and Kurtz, 1945) par une solution de fluor d'ammonium $\left(\mathrm{NH}_{4} \mathrm{~F}\right)$ 0,03 $\mathrm{M}$ et d'acide chlorhydrique 0,025 $\mathrm{M}$ dans un rapport sol/solution de $1 / 7$. Le P extrait est déterminé par colorimétrie.

\section{Analyses statistiques}

Les données collectées et saisies sur Excel ont été soumises à une analyse de variance (ANOVA) avec le logiciel Genstat version 9.2. Les moyennes ont été comparées à l'aide du test de Newman Keuls au seuil de $5 \%$, ce qui nous a permis d'établir un tableau d'analyse de variance (Tableau 2).

Tableau 1: Traitements et doses de fertilisants apportés au fonio.

\begin{tabular}{|c|c|c|c|c|c|c|}
\hline \multirow{2}{*}{ Formules de fumures } & \multicolumn{2}{|c|}{ MO } & \multicolumn{2}{|c|}{ NPK } & \multicolumn{2}{|c|}{ Urée } \\
\hline & g pot $^{-1}$ & tha $^{-1}$ & mg pot ${ }^{-1}$ & $\mathrm{~kg} \mathrm{ha}^{-1}$ & $\mathrm{mg} \mathrm{pot}^{-1}$ & $\mathrm{~kg} \mathrm{ha}^{-1}$ \\
\hline $0 \mathrm{MO}$ x $0 \mathrm{NPK}$ & & & 0 & 0 & 0 & 0 \\
\hline $0 \mathrm{MO} \times 1,5 \mathrm{NPK}$ & & & 16,88 & 46,88 & 8,44 & 23,44 \\
\hline $0 \mathrm{MO} \times 2 \mathrm{NPK}$ & 0 & 0 & 22,5 & 62,5 & 11,25 & 31,25 \\
\hline $0 \mathrm{MO} \times 2,5 \mathrm{NPK}$ & & & 28,13 & 78,13 & 14,06 & 39,06 \\
\hline $0 \mathrm{MO} \times 3 \mathrm{NPK}$ & & & 33,75 & 93,75 & 16,88 & 46,88 \\
\hline $5 \mathrm{MO} \times 0 \mathrm{NPK}$ & & & 0 & 0 & 0 & 0 \\
\hline $5 \mathrm{MO} \times 1,5 \mathrm{NPK}$ & & & 16,88 & 46,88 & 8,44 & 23,44 \\
\hline $5 \mathrm{MO} \times 2 \mathrm{NPK}$ & 1,6 & 5 & 22,5 & 62,5 & 11,25 & 31,25 \\
\hline $5 \mathrm{MO}$ x 2,5 NPK & & & 28,13 & 78,13 & 14,06 & 39,06 \\
\hline $5 \mathrm{MO} \times 3 \mathrm{NPK}$ & & & 33,75 & 93,75 & 16,88 & 46,88 \\
\hline $10 \mathrm{MO}$ x 0 NPK & & & 0 & 0 & 0 & 0 \\
\hline $10 \mathrm{MO} \times 1,5 \mathrm{NPK}$ & & & 16,88 & 46,88 & 8,44 & 23,44 \\
\hline $10 \mathrm{MO} \times 2 \mathrm{NPK}$ & 3,6 & 10 & 22,5 & 62,5 & 11,25 & 31,25 \\
\hline $10 \mathrm{MO}$ x 2,5 NPK & & & 28,13 & 78,13 & 14,06 & 39,06 \\
\hline $10 \mathrm{MO}$ x $3 \mathrm{NPK}$ & & & 33,75 & 93,75 & 16,88 & 46,88 \\
\hline
\end{tabular}

Tableau 2: Analyse de variance des différents paramètres mesurés.

\begin{tabular}{|c|c|c|c|c|c|c|c|c|c|c|c|c|c|c|}
\hline \multirow{2}{*}{$\begin{array}{l}\text { Sources de } \\
\text { variation }\end{array}$} & \multicolumn{2}{|c|}{ pH } & \multicolumn{2}{|c|}{ MO } & \multicolumn{2}{|c|}{ Pass } & \multicolumn{2}{|c|}{ BAF } & \multicolumn{2}{|c|}{ BRF } & \multicolumn{2}{|c|}{ Rdt P S } & \multicolumn{2}{|c|}{ Rdt G S } \\
\hline & F.pr & $\mathbf{S}$ & F.pr & $\mathbf{S}$ & F.pr & $\mathbf{S}$ & F.pr & $\mathbf{S}$ & F.pr & $\mathbf{S}$ & F.pr & $\mathbf{S}$ & F.pr & $\mathbf{S}$ \\
\hline MO & 0,001 & HS & 0,001 & HS & 0,001 & HS & 0,012 & $\mathrm{~S}$ & 0,052 & $\mathrm{~S}$ & $<0,001$ & HS & 0,001 & HS \\
\hline EM & 0,001 & HS & 0,073 & NS & 0,001 & HS & 0,001 & HS & 0,001 & HS & $<0,001$ & HS & 0,001 & HS \\
\hline Date & & & & & & & 0,411 & NS & 0,034 & S & & & & \\
\hline MO X EM & 0,178 & NS & 0,365 & NS & 0,112 & NS & 0,688 & NS & 0,006 & HS & 0,502 & NS & 0,668 & NS \\
\hline EM X Date & & & & & & & 0,016 & S & 0,578 & NS & & & & \\
\hline
\end{tabular}

$\mathrm{MO}=$ matière organique, $\mathrm{EM}=$ engrais minéral, $\mathrm{Pass}=$ phosphore assimilable, $\mathrm{BAF}=$ biomasse aérienne du fonio, $\mathrm{BRF}=$ biomasse racinaire du fonio, Rdt P S= rendement paille du sorgho, Rdt G S= rendement grains du sorgho. 


\section{RESULTATS}

Effets des traitements sur les paramètres chimiques du sol

Le Tableau 3 présente l'évolution des caractéristiques chimiques du sol mesurées en fonction des combinaisons. On peut observer des variations nettes de ces dernières lorsqu'on passe de la fumure minérale exclusive à celles organo-minérales avec 5 tonnes de MO puis avec 10 tonnes. En effet, les pH sont beaucoup plus bas (faiblement acides) sous fertilisation minérale exclusive. De même, les taux de MO et de phosphore assimilable sont plus faibles avec les apports minéraux exclusifs. En revanche, les apports organiques (5 et $10 \mathrm{tha}^{-1}$ ) ont permis de relever le niveau des trois paramètres. Ainsi, le $\mathrm{pH}$ moyen qui est de 5,68 pour les vases à engrais minéraux exclusifs, passe à 6,61 et 7,14 respectivement avec les apports de 5 et $10 \mathrm{tha}^{-1}$ de MO. Les taux moyens de MO sont passés de $0,81 \%$ des vases à fertilisation minérale uniquement à $1,08 \%$ et $1,26 \%$ dans les vases ayant reçu $5 \mathrm{t}$ $\mathrm{ha}^{-1}$ et $10 \mathrm{t} \mathrm{ha}^{-1}$ de MO respectivement. Il en est de même pour le phosphore assimilable qui a augmenté de $21 \%$ et $34 \%$ lorsqu'on applique 5 et $10 \mathrm{t} \mathrm{ha}^{-1}$ de $\mathrm{MO}$ respectivement. En somme, toutes les formules organominérales, bien que n'ayant pas amélioré significativement les paramètres chimiques du sol (Tableau 2), ont permis de relever le niveau de chacun des éléments. Aussi, de légères fluctuations apparaissent au sein des combinaisons en fonction des doses de NPK.

Effets de l'adjonction de la matière organique au NPK en micro dose sur la biomasse du fonio

La biomasse du fonio est présentée sur le Tableau 4. Il ressort de ce tableau que les combinaisons n'ont pas eu d'effets significativement différents sur la biomasse aérienne. Toutefois, l'ensemble des traitements l'a amélioré comparativement au témoin sans apport de fertilisants et toutes les combinaisons ont enregistré des valeurs de biomasse plus élevées que le NPK et la matière organique non combinés. La biomasse racinaire et celle totale ont par contre été significativement améliorées par les fortes doses de NPK $\left(3 ; 2,5\right.$ et 2 g poquet $\left.^{-1}\right)$ sans apport organique contrairement à nos attentes. En effet, on s'attendrait plutôt à une plus grande production avec les combinaisons dont l'interaction (NPK-matière organique) permettrait une plus grande disponibilité des éléments nutritifs qu'avec chacun des fertilisants apporté séparément.

\section{Effets des dates d'apport du NPK sur la biomasse du fonio}

L'effet des dates d'application du NPK sur la production de biomasse du fonio est représenté sur la Figure 1. On se rend compte que l'apport du NPK à différentes dates n'a pas influencé significativement la production de la biomasse du fonio. En effet, quelle que soit la dose considérée, la production de la biomasse est similaire aux trois dates d'apport du NPK (Figure 1). Par ailleurs, on note d'une manière générale une augmentation proportionnelle de la biomasse à celle de la dose de NPK apportée.

Effets de la fertilisation par micro-dose sur la production du sorgho

La croissance des plants du sorgho, les rendements paille et grains sont présentés sur les Figures 2, 3 et 4.

Les hauteurs moyennes des plants sont de $36,5 \mathrm{~cm}, 40,8 \mathrm{~cm}, 42,2 \mathrm{~cm}, 45,4 \mathrm{~cm}$ et 55,3 $\mathrm{cm}$ pour les doses de NPK seul (Figure 2). Les 
fumures mixtes $\left(\mathrm{NPK}+5 \mathrm{t} \mathrm{ha}^{-1}\right.$ de matière organique) ont donné des valeurs plus élevées que les fumures minérales exclusives (76,3 $\mathrm{cm}, 105,8 \mathrm{~cm}, 89,9 \mathrm{~cm}, 94,9 \mathrm{~cm}$ et $96,4 \mathrm{~cm}$ ). Les combinaisons de NPK avec $10 \mathrm{t} \mathrm{ha}^{-1}$ de matière organique ont enregistré des hauteurs moyennes plus importantes que les précédentes $(93,5 \mathrm{~cm}, 125,8 \mathrm{~cm}, 80,9 \mathrm{~cm}$, $131,2 \mathrm{~cm}$ et $121,5 \mathrm{~cm})$. Comparées au témoin absolu, toutes les doses de NPK combinées ou non à la MO ont eu des effets positifs sur la croissance des plants. Toutefois, les traitements avec le NPK seul ont donné des croissances similaires à celle du témoin à la probabilité de $5 \%$.

En revanche, les apports combinés de fumier et du NPK améliorent de façon significative la croissance du sorgho comparativement aux fumures minérales exclusives.

On distingue cinq groupes de combinaisons. Les combinaisons les plus performantes sur le développement végétatif sont: $10 \mathrm{t} \mathrm{ha}^{-1}$ de $\mathrm{MO}+2,5 \mathrm{~g}_{\text {poquet }}{ }^{-1}$ de NPK $(131,2 \mathrm{~cm}), 10 \mathrm{t} \mathrm{ha}^{-1}$ de $\mathrm{MO}+1,5 \mathrm{~g} \mathrm{poquet}^{-1}$ de NPK $(125,8 \mathrm{~cm}), 10 \mathrm{t} \mathrm{ha}^{-1} \mathrm{de} \mathrm{MO}+3 \mathrm{~g}$ poquet $^{-1}$ de NPK $(121,5 \mathrm{~cm}), 5 \mathrm{t} \mathrm{ha}^{-1} \mathrm{de} \mathrm{MO}+$ $1,5 \mathrm{~g}^{\text {poquet }}{ }^{-1}$ de NPK $(105,8 \mathrm{~cm})$ et $5 \mathrm{t} \mathrm{ha}^{-1} \mathrm{de}$ $\mathrm{MO}+3 \mathrm{~g} \mathrm{poquet}^{-1}$ de NPK $(96,4 \mathrm{~cm})$. Les plus faibles valeurs de croissance sont obtenues avec les doses de NPK seul. La combinaison de $10 \mathrm{t} \mathrm{ha}^{-1}$ de matière organique avec 2 g poquet $^{-1}$ de NPK a eu un effet plutôt dépressif sur la croissance. D'une manière générale, on observe une grande variabilité dans la croissance des plants issus du même traitement à ce stade avec un coefficient de variation de $15,5 \%$.

Les rendements grains obtenus ont été faibles par rapport à la moyenne en milieu paysan $\left(1,7 \mathrm{t} \mathrm{ha}^{-1}\right)$. Ils se situent entre 13,3 et 32,9 gpot $^{-1}$ soit 415,6 à $1027 \mathrm{~kg} \mathrm{ha}^{-1}$ pour le rendement en paille et entre 4,9 et 30,1 gpot $^{-}$ ${ }^{1}$ soit 152,2 à $941,3 \mathrm{~kg} \mathrm{ha}^{-1}$ pour le rendement en grains. Les plus grandes valeurs au niveau de la paille (Figure 3) sont enregistrées avec les combinaisons de $5 \mathrm{t} \mathrm{ha} \mathrm{ha}^{-1} \mathrm{MO}+3$ gpoquet $^{-1}$ de NPK $\left(32,9\right.$ gpot $\left.^{-1}\right), 10 \mathrm{t} \mathrm{ha}^{-1} \mathrm{de}$

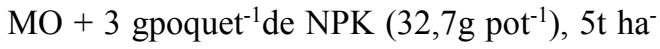
${ }^{1}+2,5$ gpoquet $^{-1}\left(32,5\right.$ g pot $\left.^{-1}\right), 10 \mathrm{tha}^{-1} \mathrm{de}_{\mathrm{MO}}$ +2 gpoquet $^{-1} \mathrm{de}^{\mathrm{NPK}}\left(32 \mathrm{gpot}^{-1}\right)$ et $10 \mathrm{t} \mathrm{ha} \mathrm{de}^{-1} \mathrm{de}$ $\mathrm{MO}+2,5$ g poquet $^{-1}$ de NPK $\left(31,4\right.$ g pot $\left.^{-1}\right)$. Sur le rendement grains (Figure 4), les combinaisons de $10 \mathrm{t} \mathrm{ha}^{-1}$ avec $3 \mathrm{~g} ; 2,5 \mathrm{~g} ; 1,5$ $\mathrm{g} ; 2 \mathrm{~g}$ et de $5 \mathrm{t} \mathrm{ha}^{-1}$ avec $3 \mathrm{~g}$ et $2 \mathrm{~g}$ sont les plus performantes $\left(30,1\right.$ gpot $^{-1}, 29,4 \mathrm{~g}$ pot $^{-1}$, 23,1 gpot $^{-1}, 22$ gpot $^{-1}, 21,8$ gpot $^{-1}$ et 21,2 gpot $^{-}$ ${ }^{1}$ respectivement). Les combinaisons de $5 \mathrm{t} \mathrm{ha}^{-1}$ et $10 \mathrm{t} \mathrm{ha}^{-1} \mathrm{de}$ MO avec le NPK ont respectivement amélioré le rendement paille de $27 \%$ et $127 \%$ et celui des grains de $50 \%$ et $87 \%$ comparativement à la fumure minérale seule. Comparativement à la fumure organique seule, on enregistre des taux d'accroissement de $55 \%$ et $17 \%$ sur le rendement paille et $48 \%$ et $42 \%$ sur le rendement grains avec respectivement $5 \mathrm{t} \mathrm{ha}^{-1}$ et $10 \mathrm{tha}^{-1}$ de fumier. Les traitements ne sont cependant pas statistiquement différents. 
Tableau 3: Effets de l'adjonction de la matière organique au NPK en micro dose sur les caractéristiques chimiques du sol.

\begin{tabular}{lccc}
\hline Traitements & pH eau & MO $(\mathbf{\%})$ & $\begin{array}{c}\text { P assimilable } \\
\text { (mg/kg de sol) }\end{array}$ \\
\hline $0 \mathrm{t} \mathrm{MO}+$ 0g par poquet de NPK & 5,69 & 0,56 & 5,05 \\
0t MO + 1,5g par poquet de NPK & 5,71 & 0,90 & 5,06 \\
0t MO + 2g par poquet de NPK & 5,69 & 0,81 & 5,18 \\
0t MO + 2,5g par poquet de NPK & 5,64 & 0,85 & 5,18 \\
0t MO + 3g par poquet de NPK & 5,66 & 0,91 & 5,63 \\
5t MO + 0g par poquet de NPK & 6,74 & 1,06 & 6,19 \\
5t MO + 1,5g par poquet de NPK & 6,58 & 1,09 & 6,49 \\
5t MO + 2g par poquet de NPK & 6,56 & 1,06 & 6,31 \\
5t MO + 2,5g par poquet de NPK & 6,61 & 1,08 & 6,98 \\
5t MO + 3g par poquet de NPK & 6,57 & 1,11 & 7,06 \\
10t MO + 0g par poquet de NPK & 7,30 & 1,2 & 6,82 \\
10t MO + 1,5g par poquet de NPK & 7,12 & 1,26 & 8,06 \\
10t MO + 2g par poquet de NPK & 7,11 & 1,31 & 8,11 \\
10t MO + 2,5g par poquet de NPK & 7,12 & 1,28 & 8,21 \\
10t MO + 3g par poquet de NPK & 7,04 & 1,26 & 8,45 \\
LSD (5 \%) & $\mathbf{0 , 1 2 1 2}$ & $\mathbf{0 , 1 8 8 2}$ & $\mathbf{0 , 7 5 1 3}$ \\
Probabilité & $\mathbf{0 , 1 7 8}$ & $\mathbf{0 , 3 6 5}$ & $\mathbf{0 , 1 1 2}$ \\
Signification & $\mathbf{N S}$ & $\mathbf{N S}$ & $\mathbf{N S}$ \\
\hline
\end{tabular}

$\mathrm{NS}=$ non significatif.

Tableau 4: Effets de la combinaison matière organique - NPK en microdose sur la biomasse du fonio.

\begin{tabular}{lccc}
\hline Traitements & $\begin{array}{c}\text { Biomasse } \\
\text { aérienne }\end{array}$ & $\begin{array}{c}\text { Biomasse } \\
\text { racinaire } \\
\text { mg/pot }\end{array}$ & $\begin{array}{c}\text { Biomasse } \\
\text { totale }\end{array}$ \\
\hline 0 t/ha MO + 0 g par poquet de NPK & 167 & $110,7 \mathrm{f}$ & $277 \mathrm{~g}$ \\
0 t/ha MO + 1,5 g par poquet de NPK & 519 & $294,3 \mathrm{cde}$ & $813 \mathrm{e}$ \\
0 t/ha MO + 2 g par poquet de NPK & 657 & $436,6 \mathrm{ab}$ & $1093 \mathrm{bc}$ \\
0 t/ha MO + 2,5 g par poquet de NPK & 660 & $467,4 \mathrm{ab}$ & $1127 \mathrm{abc}$ \\
0 t/ha MO + 3 g par poquet de NPK & 702 & $499,8 \mathrm{a}$ & $1202 \mathrm{ab}$ \\
5 t/ha MO + 0 g par poquet de NPK & 353 & $184,1 \mathrm{ef}$ & $537 \mathrm{f}$
\end{tabular}




\begin{tabular}{lccc}
5 t/ha MO + 1,5 g par poquet de NPK & 652 & $357,5 \mathrm{c}$ & $1010 \mathrm{~cd}$ \\
$5 \mathrm{t} / \mathrm{ha} \mathrm{MO} \mathrm{+} \mathrm{2} \mathrm{g} \mathrm{par} \mathrm{poquet} \mathrm{de} \mathrm{NPK}$ & 754 & $332,6 \mathrm{c}$ & $1087 \mathrm{bcd}$ \\
$5 \mathrm{t} / \mathrm{ha} \mathrm{MO} \mathrm{+} \mathrm{2,5} \mathrm{g} \mathrm{par} \mathrm{poquet} \mathrm{de} \mathrm{NPK}$ & 765 & $336,1 \mathrm{c}$ & $1101 \mathrm{abc}$ \\
$5 \mathrm{t} / \mathrm{ha} \mathrm{MO} \mathrm{+} \mathrm{3} \mathrm{g} \mathrm{par} \mathrm{poquet} \mathrm{de} \mathrm{NPK}$ & 892 & $364,1 \mathrm{c}$ & $1256 \mathrm{a}$ \\
$10 \mathrm{t} / \mathrm{ha} \mathrm{MO} \mathrm{+} \mathrm{0} \mathrm{g} \mathrm{par} \mathrm{poquet} \mathrm{de} \mathrm{NPK}$ & 408 & $229,6 \mathrm{de}$ & $637 \mathrm{f}$ \\
$10 \mathrm{t} / \mathrm{ha} \mathrm{MO} \mathrm{+} \mathrm{1,5} \mathrm{g} \mathrm{par} \mathrm{poquet} \mathrm{de} \mathrm{NPK}$ & 585 & $337,6 \mathrm{c}$ & $923 \mathrm{de}$ \\
$10 \mathrm{t} / \mathrm{ha} \mathrm{MO} \mathrm{+} \mathrm{2} \mathrm{g} \mathrm{par} \mathrm{poquet} \mathrm{de} \mathrm{NPK}$ & 771 & $331,4 \mathrm{c}$ & $1102 \mathrm{abc}$ \\
10 t/ha MO + 2,5 g par poquet de NPK & 758 & $282,4 \mathrm{cde}$ & $1041 \mathrm{~cd}$ \\
10 t/ha MO + 3 g par poquet de NPK & 830 & $384,1 \mathrm{bc}$ & $1214 \mathrm{ab}$ \\
\hline Probabilité P & $\mathbf{0 , 6 8 8}$ & $\mathbf{0 , 0 0 6}$ & $\mathbf{0 , 0 2 7}$ \\
Signification & $\mathbf{N S}$ & $\mathbf{H S}$ & $\mathbf{S}$ \\
\hline
\end{tabular}

Les moyennes affectées de la même lettre ne sont pas significativement différentes au seuil de $5 \%$. NS = non significatif, $\mathrm{HS}=$ hautement significatif, $\mathrm{S}=$ significatif.

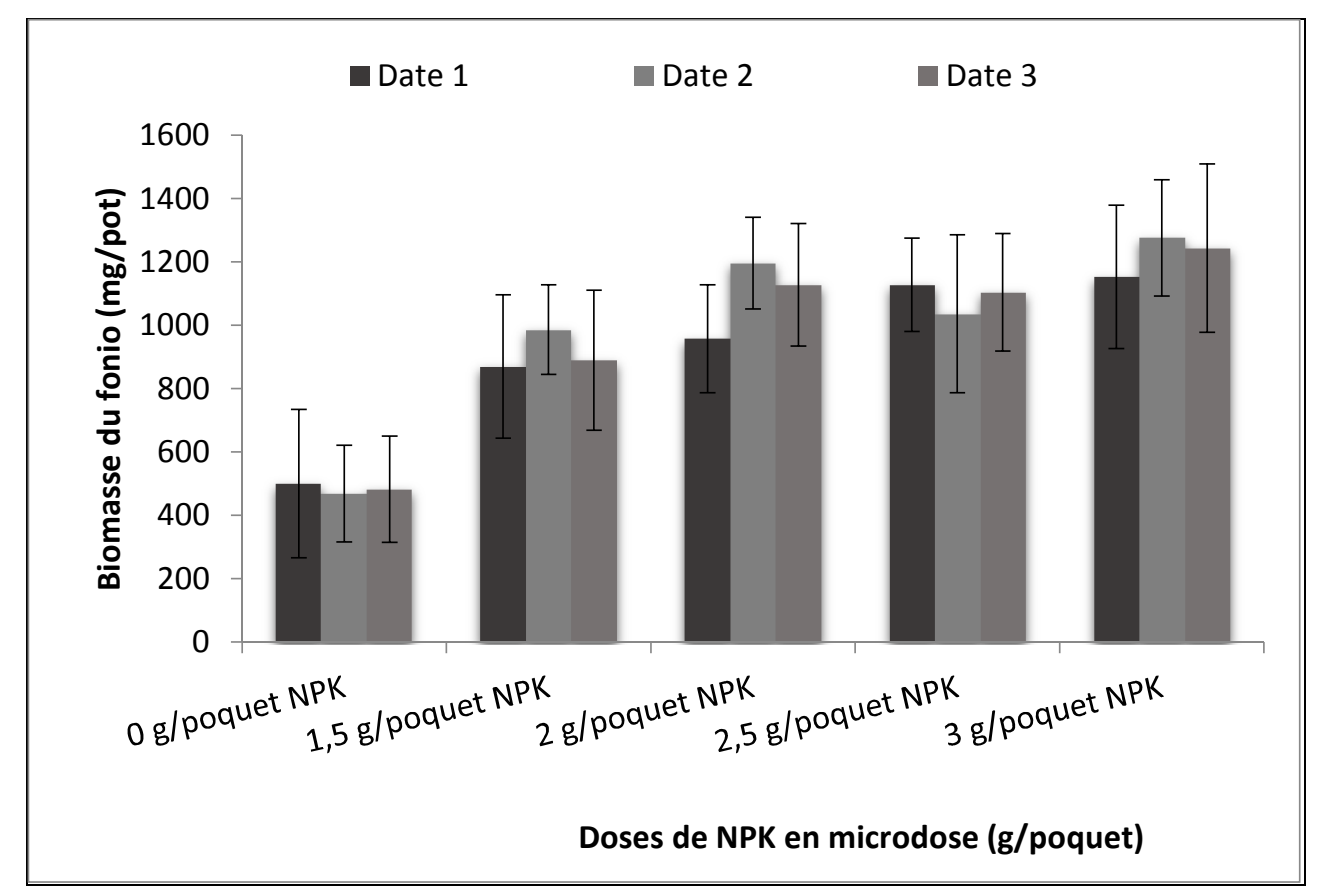

Figure 1: Effets des dates d'application du NPK sur la biomasse totale du fonio. 


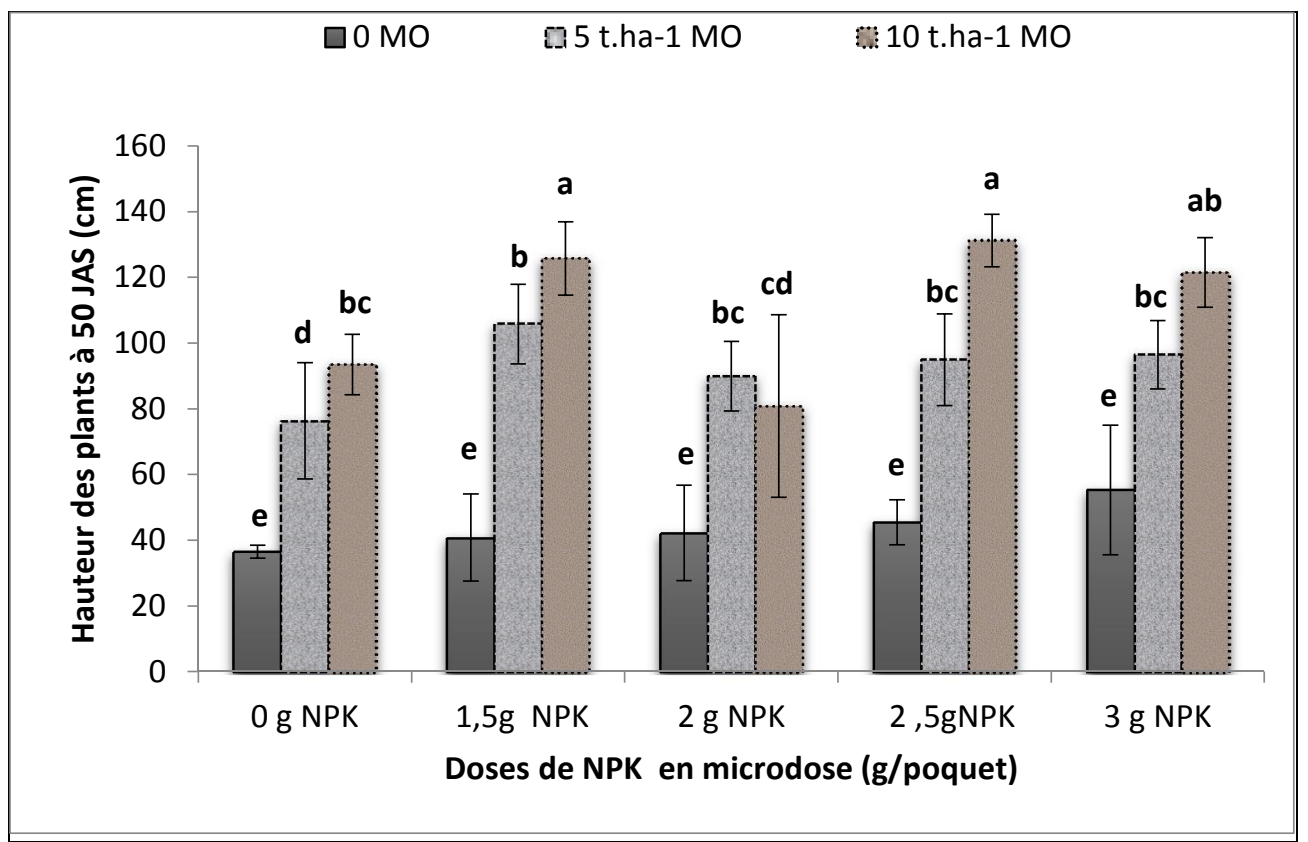

Figure 2: Effets des combinaisons de matière organique et de NPK en microdose sur la croissance du sorgho à 50 jours après semis.

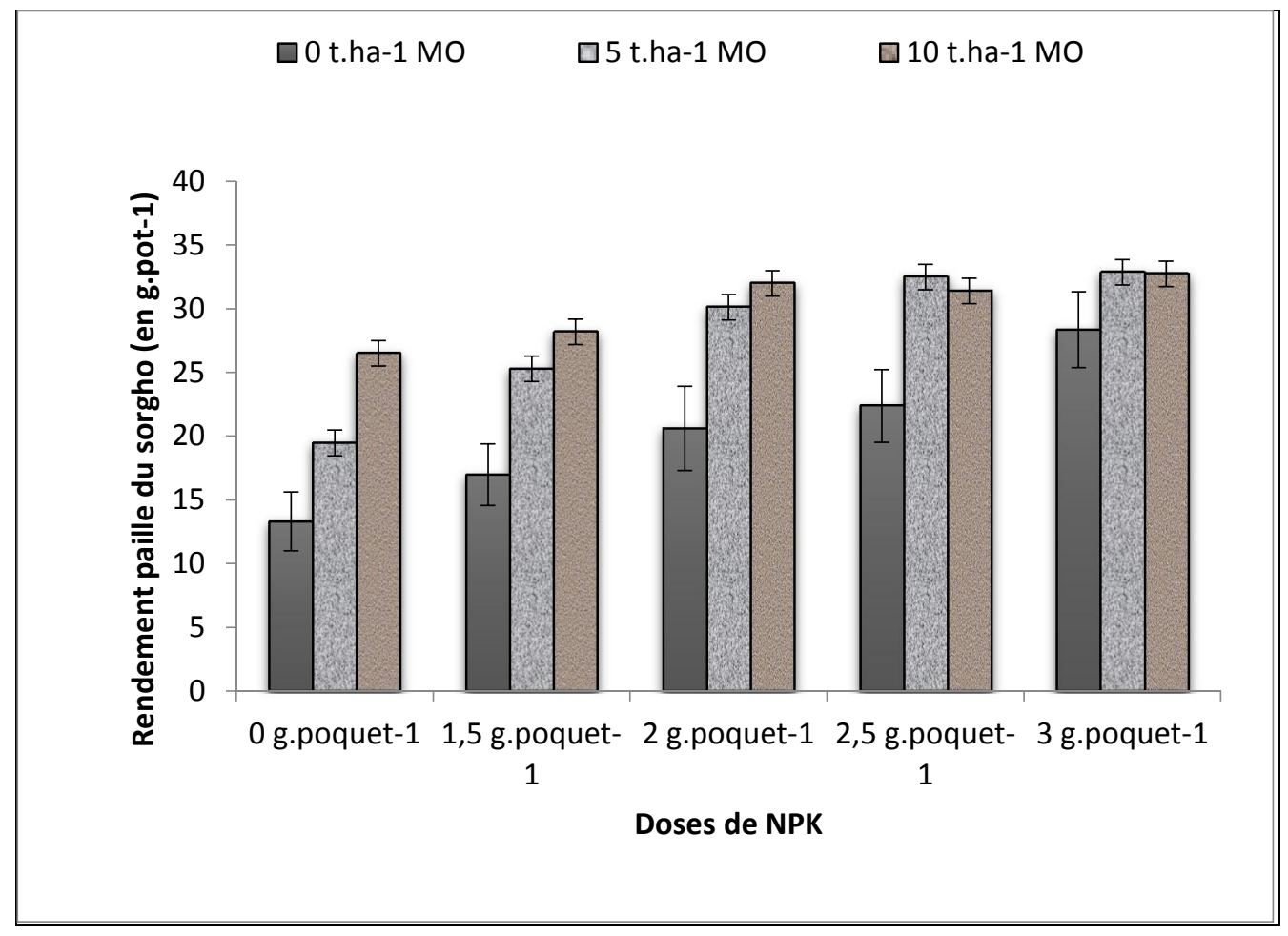

Figure 3: Effets des apports organo-minéraux sur le rendement paille du sorgho. 


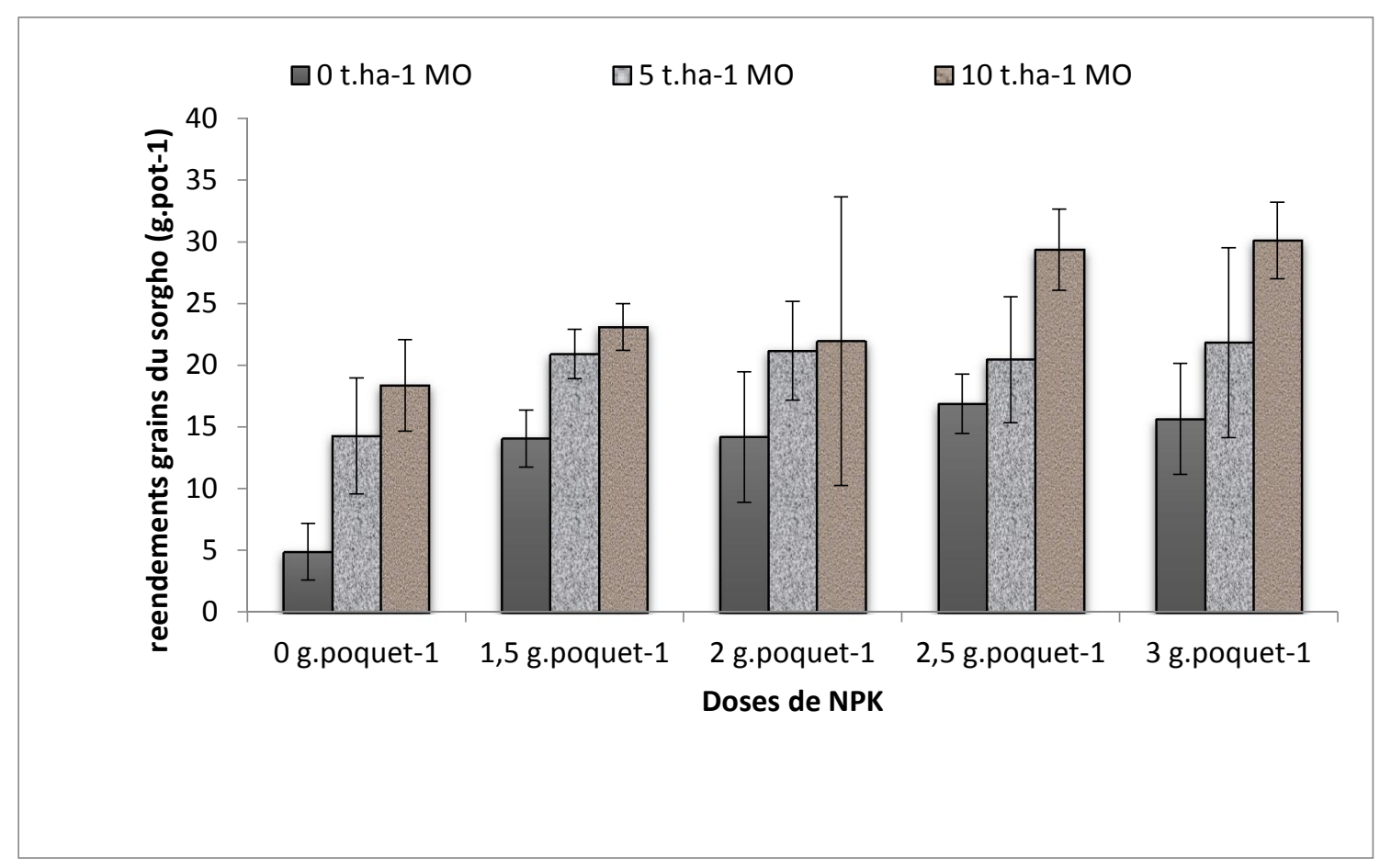

Figure 4: Effets des apports organo-minéraux sur le rendement grain du sorgho.

\section{DISCUSSION}

Les faibles valeurs initiales des caractéristiques chimiques du sol $(\mathrm{pH}$, matière organique et phosphore assimilable) ont augmenté avec l'application du fumier. Les faibles teneurs du sol en ces éléments avec la fertilisation minérale exclusive pourraient s'expliquer par les petites doses d'engrais chimiques apportées qui ont été directement prélevées par les cultures. Les variations observées avec les apports organiques et organo-minéraux seraient dues aux fumures organiques. En effet, le fumier est une source importante de matière organique et d'éléments nutritifs pour le sol tels que le phosphore. C'est également une source d'énergie et d'aliments pour les microorganismes du sol favorisant ainsi la disponibilité des éléments nutritifs (Yaméogo et al., 2013). De ce fait, son apport permet d'augmenter la capacité nutritive du sol, d'où le rehaussement du niveau des nutriments disponibles en l'occurrence le phosphore assimilable comme précédemment observé par Akanza et Yoro (2003 et 2014) en Côte d'Ivoire. Ciesielski et al. (2008) ont remarqué une influence prépondérante du carbone organique sur l'évolution du $\mathrm{pH}$ du sol suite à un apport de carbonate de calcium. Bassala et al. (2008) et Akanza et al. (2014), ont également noté qu'un apport de fumier permet de résoudre les problèmes de chutes de teneur en matière organique et d'acidification. 
L'amélioration de la biomasse aérienne $\mathrm{du}$ fonio et des rendements du sorgho suite aux apports organo-minéraux montre une fois de plus le rôle primordial des engrais minéraux et des amendements organiques apportés de façon conjointe dans la production agricole. Leurs combinaisons ont fourni les meilleurs résultats. Des résultats similaires ont été trouvés par Akanza et Yoro (2003) et Akanza et al. (2014) qui ont montré que l'application conjointe de la fumure minérale et de la fumure organique augmentait la production du maïs et du riz. En effet, la fumure organique améliore l'efficience des engrais minéraux (Akanza et al., 2014). Ces combinaisons rendraient plus disponibles les éléments de croissance des plants tel que le phosphore comme l'indique nos analyses chimiques. De même, les résultats de Soma (2010) ont démontré cela en comparant différentes combinaisons de fumure organique avec la fumure minérale à la fumure minérale exclusive. Par ailleurs, nos résultats confirment la dose vulgarisée de 5 tha $^{-1}$ tous les deux ans de matière organique, proposée par la Recherche au Burkina Faso. Contrairement à ces résultats, Kanfany (2010) a noté en milieu réel au Sénégal que l'interaction engrais minéral-matière organique n'a pas induit d'effets significatifs sur la biomasse du fonio. Sur la biomasse racinaire et totale du fonio, seules les fortes doses de NPK ont donné les meilleurs résultats, quoique non significatifs, par rapport aux combinaisons contrairement aux nombreux résultats de recherche qui ont indiqué des résultats plus intéressants avec les combinaisons qu'avec un seul type de fertilisant. Ce résultat pourrait probablement s'expliquer par les blocs de terre qui seraient restés entre les racines lors de l'extraction de celle-ci des vases. La terre était en effet peu friable dans les vases sans matière organique si bien qu'elle s'est plus adhérée aux racines que dans les autres pots. Il pourrait également s'agir d'erreurs de mesures comme l'ont souligné Akanza et al. (2014). En tout état de cause, les résultats obtenus montrent que les doses optimales d'apport d'engrais NPK encadrent la dose de $2 \mathrm{~g}$ par poquet, en cours de vulgarisation (Tabo et al., 2007 ; Palé et al., 2009).

Les dates d'apport du NPK sur le fonio n'ont pas eu d'effets significatifs sur la production de biomasse. Cela signifierait que la date de semis peut se situer indifféremment entre le jour du semis et 15 jours après le semis. En effet, jusqu'à cette période, les besoins nutritifs des plantes sont importants et donc un apport en ce moment est bien indiqué pour leur croissance. C'est d'ailleurs la fourchette de temps qu'a suggéré UA/SAFGRAD (2010). Ces résultats confirment ceux de Hayashi et al. (2008) qui ont également montré qu'un apport de l'engrais minéral 21 jours après semis n'influe pas sur les rendements des cultures.

\section{Conclusion}

La présente étude a permis d'évaluer les effets de l'adjonction de trois doses de fumier à cinq niveaux de NPK appliqué en micro-dose sur la biomasse du fonio, les rendements du sorgho et les caractéristiques chimiques du sol. En outre, un effet date d'apport du NPK a été évalué. Il ressort de cette expérimentation que: les différentes doses de matière organique ( 5 et 10$)$ et de l'engrais minéral $(1,5 ; 2 ; 2,5$ et 3$)$ apportées séparément permettent d'améliorer la production de biomasse aérienne et totale du fonio ainsi que les rendements du sorgho; les 
combinaisons engrais minéral-fumure organique ont enregistré de meilleurs rendements comparativement aux apports dissociés de chacun de ces fertilisants. Les doses optimales d'apport d'engrais NPK encadrent la dose de $2 \mathrm{~g}$ par poquet, en cours de vulgarisation. Les résultats confirment également la dose vulgarisée de 5 tha $^{-1}$ tous les deux ans, de matière organique, proposée par la Recherche au Burkina Faso. Le pH, les teneurs en matière organique et en phosphore assimilable du sol ont connu une hausse grâce aux apports combinés du NPK et du fumier; en définitive, la bonne gestion de la fertilité du sol et l'amélioration des productions agricoles passent nécessairement par l'utilisation conjointe de la fumure organique et des engrais minéraux. L'apport du NPK en microdose peut se faire indifféremment entre le jour $\mathrm{du}$ semis et 15 jours après semis sans compromettre la production.

\section{CONFLIT D'INTERETS}

Les auteurs déclarent qu'il n'y a aucun conflit d'intérêt.

\section{CONTRIBUTIONS DES AUTEURS}

BO a participé à la conception du dispositif expérimental et l'écriture de l'article; IS et MBP ont participé aux analyses statistiques et à la correction du manuscrit ; FL, SJBT et PMS ont participé à la correction du protocole de recherche, au suivi des travaux de collecte de données sur le terrain et à la correction du projet d'article.

\section{REMERCIEMENTS}

Nous adressons nos sincères remerciements à toutes les personnes ayant contribué sur le plan technique à faire aboutir cette étude.

\section{REFERENCES}

AFNOR. 1981. Détermination du $\mathrm{pH}$. (Association Française de Normalisation). nf iso 103 90. In Afnor Qualité des Sols. AFNOR : paris ; 339-348.

Akanza KP, Sanogo S, Kouakou CK, N'Da HA, Yao-Kouamé A. 2014. Effets de la fertilisation sur la fertilité des sols et les rendements: incidence sur le diagnostic des carences du sol. Rev. Ivoir. Sci. Technol., 24: 299-315. DOI : http:// www.revist.ci

Akanza PK, Yoro G. 2003. Effets synergiques des engrais minéraux et de la fumure de volaille dans l'amélioration de la fertilité d'un sol ferrallitique de l'ouest de la côte d'ivoire. Agronomie Africaine., 15(3): 135-144. DOI : http: //dx.doi.org/10.4314/aga.v15i3.1631

Bado BV. 2002. Rôle des légumineuses sur la fertilité des sols ferrugineux tropicaux des zones guinéenne et soudanienne du Burkina Faso. Thèse de Doctorat, Université Laval Québec, p.184.

Bagayoko M, Maman N, Palé S, Sirifi S, Taonda SJB, Traore S, Mason SC. 2011. Microdose and $\mathrm{N}$ and $\mathrm{P}$ fertilizer application rates for pearl millet in West Africa. African Journal of Agricultural Research, 6(5): 1141-1150. DOI : 10.5897/AJAR10.711

Barro A, Zougmoré R, Sedogo PM. 2009. Evaluation de la faisabilité de trois types de travail du sol: application du modèle Sarra dans le plateau central du Burkina Faso. Sécheresse, 4: 338-45. DOI: http://www.jle.com/10.1684/sec.2009.020 2

Buerkert A, Bationo A, Piepho HP. 2001. Efficient phosphorus application strategies for increased crop production in Sub- 
Saharan West Africa. Field Crops

Research, 72: 1-15. DOI:

http://www.sciencedirect.com/science/artic le/pii/S0378429001001666.

Coulibaly A, Aune Jens B, Sissoko P. 2010.

Etablissement des cultures vivrières dans les znones sahélienne et soudano sahélienne du Mali. GCoZA Rapport $\mathrm{N}^{\circ} 60.65 \mathrm{p}$.

Hayashi K, Abdoulaye T, Gerard B, Bationo A.2007. Evaluation of application timing in fertilizer micro-dosing technology on millet production in Niger, West Africa. Nutrient Cycling and Agroecosystem, 80, 257-265. DOI: 10.1007/s10705-007-91413.

Lompo F. 2009. Effets induits des modes de gestion de la fertilité sur les états du phosphore et la solubilisation des phosphates naturels dans deux sols acides $\mathrm{du}$ Burkina Faso. Thèse de doctorat. Université de Cocody, p 200.

Olina Bassala JP, M'Biandoun M, Ekorong JA, Asfom P. 2008. Evolution de la fertilité des sols dans un système cotonnier-céréales au Nord Cameroun: diagnostic et perspectives. Tropicultura, 26(4): 240-245. DOI: http:// www.tropicultura.org/text/v26n4.pdf.

Masse D. 2007. Changements d'usage des terres dans les agro-systèmes d'Afrique Sub-saharienne. Propriétés des sols et dynamique des matières organiques. Mémoire d'habilitation à diriger des recherches. Institut National Polytechnique de Toulouse. Ecole Nationale Supérieure Agronomique de Toulouse, p.82.

Palé S, Mason SC, Taonda SJB. 2009. Water and fertilizer influence on yield of grain sorghum varietiesproduced in Burkina Faso. S. Afr. J. Plant Soil, 26(2): 91- 97.
DOI: $\quad$ http://www.tandfonline. com/doi/pdf/10.1080/02571862.2009.1063 9939

Segda Z. 2006. Gestion de la fertilité du sol pour une production améliorée et durable du riz (Oryza sativa L.) au Burkina Faso. Cas de la plaine irriguée de Bagré. Thèse de doctorat de l'université de Ouagadougou. Option: Biologie et Ecologie Végétales, p146.

Soma M. 2010. Effet des apports répétés de diverses sources d'amendements organiques dans un sol ferrugineux tropical lessivé (Saria, Burkina Faso) sur la biodisponibilité du phosphore et la production du sorgho. Mémoire de DEA. Université Polytechnique de BoboDioulasso, p60.

Tabo R, Bationo A, Diallo Maimouna K, Hassane O, Koala S. 2006. Fertilizer microdosingfor the prosperity of smallscale farmers in the Sahel: Final report. Global Theme on Agroecosystems Report no. 23. PO Box 12404, Niamey, Niger: International Crops Research Institute for the Semi-Arid Tropics, p28.

Tabo R, Bationo A, Gerard B, Ndjeunga J, Marchal D, Amadou B, Garba M.A, Sogodogo D, Taonda JBS, Hassane O, Diallo MK, Koala S. 2007. Improving cereal productivity and farmers' income using a strategic application of fertilizers in West Africa. In Advance in Integrated Soil Fertility Management in Sub-Saharan Africa: Challenges and Opportunities, Bationo A, Waswa B, Kihara J, Kimetu J (eds). Proceeding of Afnet International Symposium 17 - 21 September 2004, Yaoundé Cameroun. Springer, 2007: 589 $-598$. 
Tabo R, Bationo A, Hassane O, Amadou B, Fosu M, Sawadogo S, Kabore, Fatondji D, Ouattara K, Abdou A, Koala S. 2008. Fertilizer microdosing for the prosperity of resource poor farmers: a success story. 269 - 278 in Humphreys E, Bayot RS. (Editors). 2009. Increasing the productivity and sustainability of rainfed cropping systems of poor smallholder farmers. Proceedings of the CGIAR Challenge Program on Water and Food International Workshop on Rainfed Cropping Systems, Tamale, Ghana, 22-25 September 2008. The CGIAR Challenge Program on Water and Food, Colombo, Sri Lanka p.311.

Twomlow S, Rohrbach D, Dimes J, Rusike J. 2008. Micro-dosing as a pathway to Africa's Green Revolution: evidence from broad-scale on-farm trials.Nutrient Cycling
Agroecosystem, 88: 3-15. DOI: doi:10.1007/s10705-008-9200-4

UA/SAFGRAD. 2010. Recherche et développement agricoles dans les zones semi-arides d'Afrique. Technologies agricoles au Burkina Faso. Recueil des technologies agricoles, p100.

Walkley A, Black JA. 1934. An examination of the Detjareff method for determining soil organic matter and a proposed modification of the chromatic acid titration method. Soil Science, 37: 29-38.

Yaméogo JT, Somé AN, Mette Lykke A, Hien M, Nacro HB. 2013. Restauration des potentialitésde sols dégradés à l'aide du zaï et des cordons pierreux à l'Ouest du Burkina Faso. Tropicultura, 31(4): 224230.http://www.tropicultura.org/text/v31n 4/224.pdf. 